\title{
Phugoid Motion for Grazing-Entry Trajectories at Near-Circular Speeds
}

\author{
Nguyen X. Vinh, ${ }^{*}$ Vincent T. Coppola ${ }^{\dagger}{ }^{\text {and L. de-Olivé Ferreira }}{ }^{\ddagger}$ \\ University of Michigan, Ann Arbor, Michigan 48109-2118
}

\begin{abstract}
We analyze the trajectory perturbations that result in phugoid oscillations during the grazing planetary entry of space vehicles starting with near-circular speed, in ballistic and equilibrium glide modes. The equations for planar entry are transformed into a dimensionless system appropriate for an analytical integration that provides accurate results in comparison with those obtained from a numerical integration of the original system. For ballistic entry, the small perturbation in the flight-path angle is a lightly damped oscillation with long period, while the perturbation in the density (altitude) increases as the speed decreases. Nevertheless, the relative change in the density is decreasing, and the phugoid in ballistic entry is termed stable in both the flight-path angle and the altitude. For glide entry, in the reference solution where there is equilibrium of forces along the normal to the flight path, both the flight-path angle and the altitude have a steady decrease along the flight path. With a slight perturbation in either the initial speed or the initial flight-path angle, or both, the trajectory variables undergo a damped oscillation about the reference trajectory. Both the damping and the frequency are obtained explicitly, and they correctly predict the phugoid motion as seen in the numerical solution.
\end{abstract}

$=$ coefficient function in Boole's method; constant of integration in the analysis of gliding entry

$a_{m}, b_{m} \quad=$ series coefficients in Boole's method

$B=$ coefficient function in Boole's method

$C=$ constant of integration in the analysis of ballistic entry

$C_{D}, C_{L} \quad=$ coefficients of drag and lift, respectively

$c \quad=$ dimensionless flight-path angle variable at entry

$f_{1}, g_{1} \quad=$ series used in the analysis of ballistic entry (altitude)

$f_{2}, g_{2} \quad=$ series used in the analysis of ballistic entry (flight-path angle)

$g \quad=$ magnitude of the acceleration due to gravity

$H \quad$ = altitude from the reference level

$h \quad=$ dimensionless altitude from the reference level

$k=$ auxiliary parameter in the analysis of ballistic entry

$L \quad$ = aerodynamic lift

$m \quad=$ vehicle's mass

$N \quad$ = number of oscillations in gliding entry

$r \quad=$ radial distance from planet's center

$S \quad=$ vehicle's characteristic area

$s \quad=$ dimensionless arc length along trajectory

$t \quad=$ time

$u \quad=$ dimensionless speed in terms of the kinetic energy

$V \quad=$ speed along the trajectory

$x \quad=$ alternate form of the dimensionless speed (negative natural logarithm of $u$ )

$=$ Chapman's altitude variable

$=$ transformed perturbation in $Y$ in ballistic entry

$=$ transformed flight-path angle variable in the analysis of gliding entry

$=$ inverse scale height

$=$ flight-path angle

$=$ independent variable in Boole's method

Received March 30, 1995; revision received Nov. 20, 1995; accepted for publication Dec. 11, 1995. Copyright (C) 1996 by the American Institute of Aeronautics and Astronautics, Inc. All rights reserved.

*Professor of Aerospace Engineering, Department of Aerospace Engineering. Member AIAA.

${ }^{\dagger}$ Assistant Professor, Department of Aerospace Engineering.

${ }^{\ddagger}$ Ph.D. Candidate, Department of Aerospace Engineering. Student Member AIAA. $\lambda$

$\mu \quad=$ transformed speed variable in the analysis of gliding entry

$=$ atmospheric density

$\rho \quad \quad=$ dimensionless flight-path angle

$\omega \quad=$ augmented lift-to-drag ratio; Eq. (52)

$\bar{\omega} \quad=$ averaged frequency of gliding phugoid mode

\section{Subscripts}

$c \quad=$ circular condition; complementary function

$f \quad=$ final condition

$0 \quad=$ reference trajectory; condition at entry; point where the solutions for the phugoid are connected

\section{Introduction}

$\mathrm{N}$ a recently revised and extended treatise on planetary entry dynamics, Regan and Anandakrishnan ${ }^{1}$ review the most significant results in the field, from the seminal works of Chapman ${ }^{2}$ and Allen and Eggers ${ }^{3,4}$ to the recent studies on aeroassist technology by a new generation of researchers. ${ }^{5}$ The general problem is currently being extended to cover the whole range of entry speeds, from suborbital flight of ballistic missiles to hyperbolic entry of spacecraft into planetary atmospheres, with control techniques implemented to optimize the various physical performance parameters. However, for the most part, the published literature addresses only specific formulations, usually based upon restrictive assumptions on the entry speed, entry angle, and range of lift-to-drag ratio for the vehicle. As examples, solutions are readily obtained for the case of ballistic entry at suborbital speed with a medium or steep angle as well as for grazing entry at supercircular speed, in which the vehicle will skip out after a shallow dip into the upper atmosphere. On the other hand, a very sensitive case has, so far, been left largely unexplored, except when considered as the final phase of the problem of orbit decay: the problem of entry at near-circular speed and a very small flight-path angle. Yet this case often happens (e.g., in the entry from low circular orbits or abort during ascent into orbit). In particular, no major effort has been made to produce stability analysis results for such case.

One of the main difficulties associated with the construction of an analytic theory for stability analysis of re-entry vehicles is the determination of the reference trajectory, since entry dynamics is a strongly unsteady, nonlinear phenomenon. Singularities in the formulation, as well as sensitivity and accuracy issues, compound the 
hardship. The first significant attempt at circumventing the problem was put forth in Loh's comprehensive work. ${ }^{6}$ Unfortunately, his construction of the solution is flawed by the introduction of an empirical step that cannot be mathematically justified. A year later, Yaroshevskii ${ }^{7}$ introduced the first mathematical solution to the problem. But this solution is still not satisfactory for ballistic grazing entry at small angles.

In this paper, the singularity inherent in the formulation for entry at near-circular speed and nearly zero flight-path angle, for both the ballistic mode and the glide mode, is removed by a normalizing transformation of the time and altitude (density) variables. Then, by introducing a small initial perturbation, the behavior of the variations in the relevant elements is analyzed and the convergence of each mode is studied. In particular, the paper introduces a new, more accurate solution for the reference trajectory than Yaroshevskii's solution. Finally, a new set of analytic solutions for the perturbed elements of the trajectory is obtained and shown to display correctly the phugoid behavior found in the numerical solutions in both the ballistic case and the glide case. Stability is assessed from the analytic solutions and confirmed by simulation of the nonlinear equations.

\section{Dimensionless Equations of Motion}

Consider Fig. 1, depicting a planetocentric inertial reference frame. With respect to this frame, the equations of motion of a nonthrusting aerospace vehicle, considered as a point mass, in planar entry into a spherical, nonrotating planetary atmosphere are

$$
\begin{gathered}
\frac{\mathrm{d} r}{\mathrm{~d} t}=V \sin \gamma \\
r \frac{\mathrm{d} \lambda}{\mathrm{d} t}=V \cos \gamma \\
\frac{\mathrm{d} V}{\mathrm{~d} t}=-\frac{D}{m}-g \sin \gamma \\
V \frac{\mathrm{d} \gamma}{\mathrm{d} t}=\frac{L}{m}-\left(g-\frac{V^{2}}{r}\right) \cos \gamma
\end{gathered}
$$

In the classical theory of low-speed, low-altitude flight, longitudinal dynamics is found to be characterized by two fundamental oscillation modes, namely, a long-period oscillation, called the phugoid, and a short-period oscillation, called the angle-of-attack mode. The phugoid consists of a trajectory mode in which the angle of attack remains nearly constant while the center of mass of the vehicle oscillates about a reference flight path, continually exchanging potential and kinetic energies. The angle-of-attack mode, on the other hand, is a pitching oscillation about the vehicle's center of mass. As a first approximation, the two modes are taken to be uncoupled, especially with respect to the phugoid mode. We introduce such a decoupling to study the phugoid in the case of grazing atmospheric entry. Accurate analytic solutions are sought for the re-entry trajectory at constant angle of attack, for both the ballistic mode $\left(C_{L}=0\right)$ and the glide mode $\left(C_{L} / C_{D} \neq 0\right)$. In each case, this trajectory will be used as the reference trajectory in the stability analysis to follow.

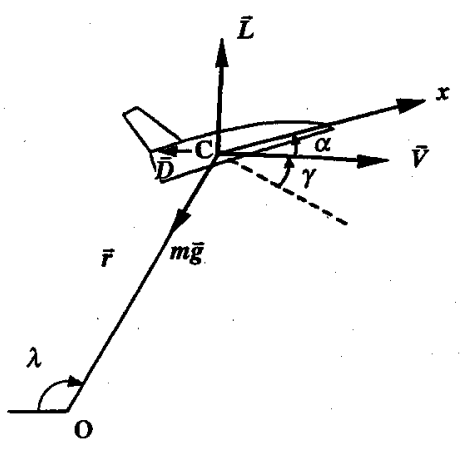

Fig. 1 Trajectory variables.
The problem of longitudinal dynamics for re-entry from a lowaltitude circular orbit is formulated as follows. At entry,

$$
\begin{array}{lrl}
t=0, \quad r=r_{0}, & \lambda=0 \\
V=V_{0}=\sqrt{g_{0} r_{0}}, & \gamma=\gamma_{0}
\end{array}
$$

We shall consider a strictly exponential atmosphere with constant value for the inverse scale height, $\beta$. Thus, in differential form,

$$
\mathrm{d} \rho=-\beta \rho \mathrm{d} r
$$

Also, the standard expressions for drag and lift will be used:

$$
D=\frac{1}{2} \rho S V^{2} C_{D}, \quad L=\frac{1}{2} \rho S V^{2} C_{L}
$$

Dimensionless variables are now introduced. The speed is nondimensionalized in terms of the kinetic energy:

$$
u=V^{2} / g_{0} r_{0}
$$

The altitude is represented by Chapman's altitude variable, which is the dimensionless atmospheric density

$$
Y=\left(\rho S C_{D} / m\right) \sqrt{\left(r_{0} / \beta\right)}
$$

and the flight-path angle is analyzed in the form of the dimensionless variable

$$
\phi=-\sqrt{\beta r_{0}} \sin \gamma
$$

All computations will be performed for the Earth, where $\beta r_{0}=900$. The motion will be parametrized using the dimensionless arc length

$$
s=\sqrt{\frac{\beta}{r_{0}}} \int V \mathrm{~d} t
$$

rather than the time $t$.

With these definitions, we have the following dimensionless equations of motion:

$$
\begin{gathered}
\frac{\mathrm{d} Y}{\mathrm{~d} s}=Y \phi \\
\frac{\mathrm{d} u}{\mathrm{~d} s}=-u Y+\frac{2}{\beta r_{0}}\left(\frac{g}{g_{0}}\right) \phi \\
\frac{\mathrm{d} \phi}{\mathrm{d} s}=-\frac{\sqrt{\beta r_{0}}}{2} Y\left(\frac{C_{L}}{C_{D}}\right) \cos \gamma+\left(\frac{g / g_{0}}{u}-\frac{r_{0}}{r}\right) \cos ^{2} \gamma
\end{gathered}
$$

Let $H$ be the altitude measured from the reference level, which here is the entry altitude, and $h$ be its normalized value, that is,

$$
H=r-r_{0}, \quad h=H / r_{0}
$$

Then,

$$
r_{0} / r=1-h+h^{2}-\cdots
$$

and, for a central Newtonian gravitational attraction,

$$
g / g_{0}=1-2 h+3 h^{2}-\cdots
$$

Since the altitude is now represented by the dimensionless density $Y$, which varies widely, and since, in the relevant terms, $h$ is of the order of $10^{-2}$, during re-entry we take $r / r_{0} \approx 1$ and $g / g_{0} \approx 1$. In addition, we consider cases for which $\gamma$ is small, so that $\cos \gamma \approx 1$. Using these approximations, the equations of motion become

$$
\begin{gathered}
\frac{\mathrm{d} Y}{\mathrm{~d} s}=Y \phi \\
\frac{\mathrm{d} u}{\mathrm{~d} s}=-u Y+\frac{2}{\beta r_{0}} \phi \\
\frac{\mathrm{d} \phi}{\mathrm{d} s}=-\frac{\sqrt{\beta r_{0}}}{2} Y\left(\frac{C_{L}}{C_{D}}\right)+\left(\frac{1}{u}-1\right)
\end{gathered}
$$


Although simple, this system of equations of motion provides accurate predictions of the altitude, speed, and flight-path angle in comparison with the values obtained from the numerical integration of the original system of Eqs. (1a-1d). Moreover, the equations are valid for any re-entry vehicle and any planetary atmosphere. It is only necessary to specify the atmosphere by choosing the value for $\beta r_{0}$ and stipulate the lift-to-drag ratio $C_{L} / C_{D}$ for the flight control. For computational purposes, we shall take $C_{L} / C_{D}$ as a constant depending on the case under study (ballistic or gliding). As a numerical comparison, we have plotted in Figs. 2 and 3 the altitude vs the speed for two ballistic entry trajectories and one gliding entry trajectory, using the results of the numerical integration of the

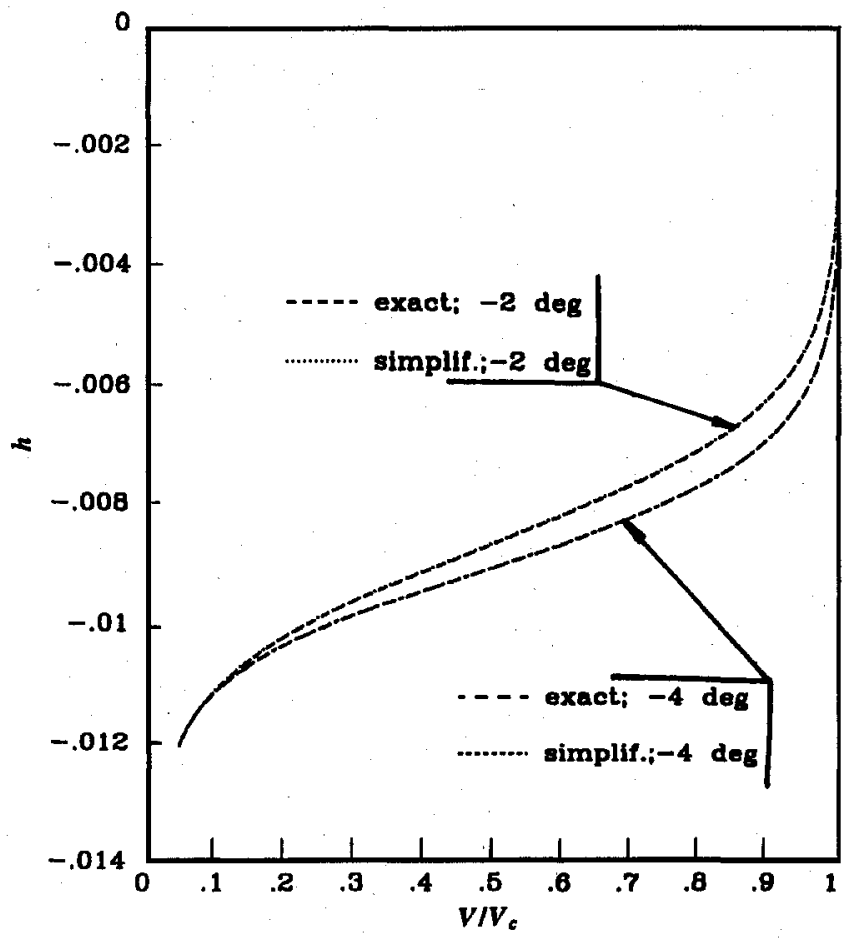

Fig. 2 Variation of the altitude as a function of the speed during ballistic entry at near-circular speed; numerical solution.

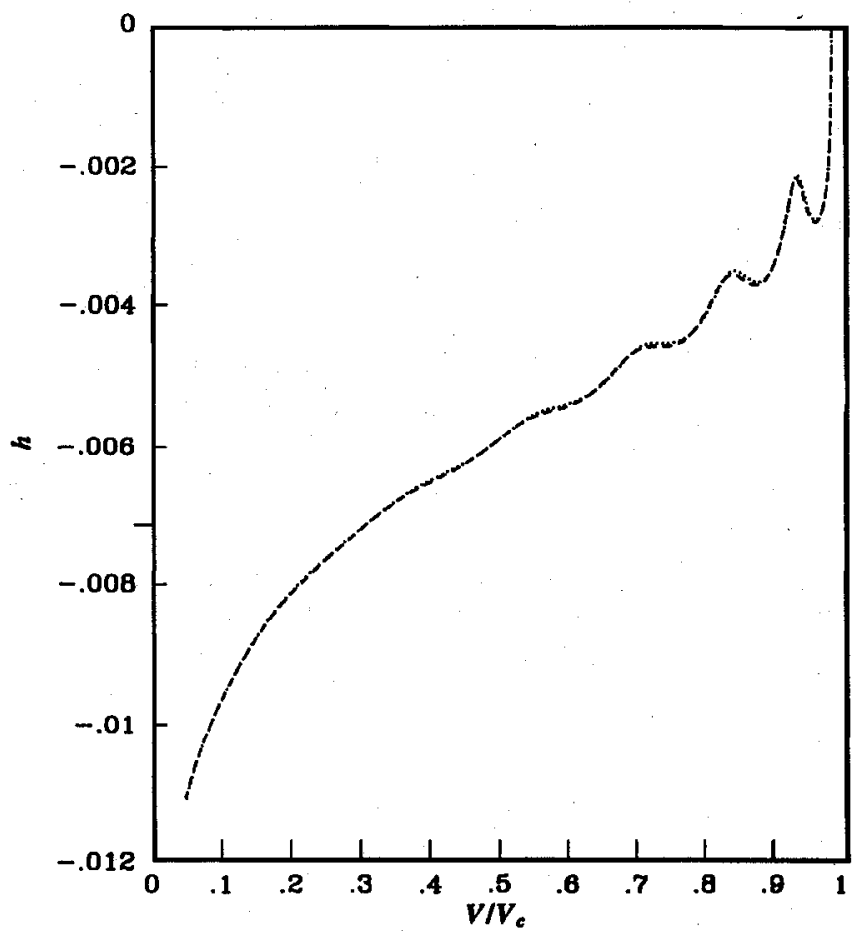

Fig. 3 Variation of the altitude as a function of the speed during gliding entry at near-circular speed; numerical solution; -0.5 deg:..-- , exact and $\cdots \cdot$, simplified. system of Eqs. (9a-9c), which is the normalized form of the original system, and the simplified system of Eqs. $(13 a-13 \mathrm{c})$. The initial altitude is such that, at entry, $Y(0)=Y_{0}=10^{-3}$. The normalized deceleration resulting from drag is $(\mathrm{d} V / \mathrm{d} t) / g_{0}=-\sqrt{ }\left(\beta r_{0}\right) Y u / 2$. At entry with circular speed and the above value for $Y_{0}$, this deceleration is $1.5 \times 10^{-2} g_{0}$, sufficient to initiate atmospheric entry. In Fig. 2 we present the plots of the dimensionless altitude vs the speed ratio $V / V_{c}$ for two typical cases of ballistic entry at $\gamma_{0}=-2$ and -4 deg. The entry speed is such that $V_{0} / V_{c}=1$. The results from the two sets of equations are in nearly perfect agreement. At a final speed ratio as low as $V_{f} / V_{c}=0.05$, which corresponds to a Mach number of 1.15 , the difference in the altitude, for the case of $\gamma_{0}=-2 \mathrm{deg}$, is $\Delta h=2.985 \times 10^{-5}$, or about $193 \mathrm{~m}$ for an entry altitude of $100 \mathrm{~km}$. In the case of $\gamma_{0}=-4 \mathrm{deg}$, the difference is only $7 \mathrm{~m}$ in $100 \mathrm{~km}$. In Fig. 3 we have the same type of plots for a glide trajectory with $C_{L} / C_{D}=1, V_{0} / V_{c}=0.98$, and $\gamma_{0}=-0.5 \mathrm{deg}$. From the same entry altitude, at a final speed of $V_{f} / V_{c}=0.05$, the difference in altitude is $149 \mathrm{~m}$.

One reason for the accuracy in using the simplified system of Eqs. (13) is that the approximation $g \approx g_{0}$ and $r \approx r_{0}$ is fully justified. In Loh's treatise, ${ }^{6}$ equations written with these simplifications are referred to as the exact equations. For all practical purposes, our approximation affects only the equation for $\phi$, Eq. (13c), as we neglect the term equivalent to $-(\mathrm{d} \phi / \mathrm{d} s) \gamma^{2} / 2$ on the right-hand side of this equation. Since, for both cases of ballistic entry and nearequilibrium glide entry, the rate of change of the flight-path angle is small, the term neglected is also small and the results are valid even for large flight-path angle.

We therefore consider the system of Eqs. (13) as the appropriate system for analyzing planar entry trajectories. The subsequent analytic solutions are compared with the numerical solutions of this system.

\section{Phugoid Motion in Ballistic Entry}

As previously mentioned, in the classical analysis of the perturbed motion in longitudinal flight of an airplane, one clearly distinguishes the phugoid motion, in which the center of mass undergoes a lightly damped oscillation about steady flight, and the angle-of-attack perturbation, which is a heavily damped oscillatory motion. Extension to orbital cruise of hypervelocity vehicles was carried out first by Etkin $^{8}$ and then by Vinh and Dobrzelecki. ${ }^{9}$ This analysis was done for the case of a steady reference trajectory (initial cruise at constant altitude with constant speed). The case for a re-entry trajectory is more difficult to analyze because an accurate analytic solution for the unsteady reference trajectory has not been available. Furthermore, even by using the existing first-order solution for the reference flight, the linearized equation for the perturbation is usually a second-order linear differential equation with varying coefficients. In general, its solution requires some preliminary transformation of both the independent and the dependent variables, and such a transformation is not always apparent. Standard power-series solutions compare poorly with the results obtained by numerical integration. The present analysis is geared toward overcoming these difficulties.

For ballistic entry, $C_{L} / C_{D}=0$. In addition, during entry, the tangential component $-g \sin \gamma$ of the gravitational acceleration is small and negligible in comparison with the deceleration due to aerodynamic drag. In other words, in Eq. (13b), the term $\left(2 / \beta r_{0}\right) \phi$ is small compared to $-u Y$; therefore, we neglect it in the analytic integration. For ballistic entry at circular speed, Eqs. (13) [neglecting $\left.\left(2 / \beta r_{0}\right) \phi\right]$ are integrated with the initial condition, Eq. (2), rewritten in terms of the dimensionless variables as

$$
Y(0)=0, \quad u(0)=1, \quad \phi(0)=-\sqrt{\beta r_{0}} \sin \gamma_{0}=c
$$

The variation of the flight-path angle as a function of the speed ratio $V / \sqrt{ }\left(g_{0} r_{0}\right)$ is plotted in Fig. 4 for entry at various initial angles $\gamma_{0}$. In the plot, the solid lines represent the numerical solutions of Eqs. (13). The analytical solution for these trajectories, plotted in dashed lines, is obtained as follows.

Define the new dimensionless speed variable $x$ as

$$
x=\ln \left(g_{0} r_{0} / V^{2}\right)=-\ln u
$$




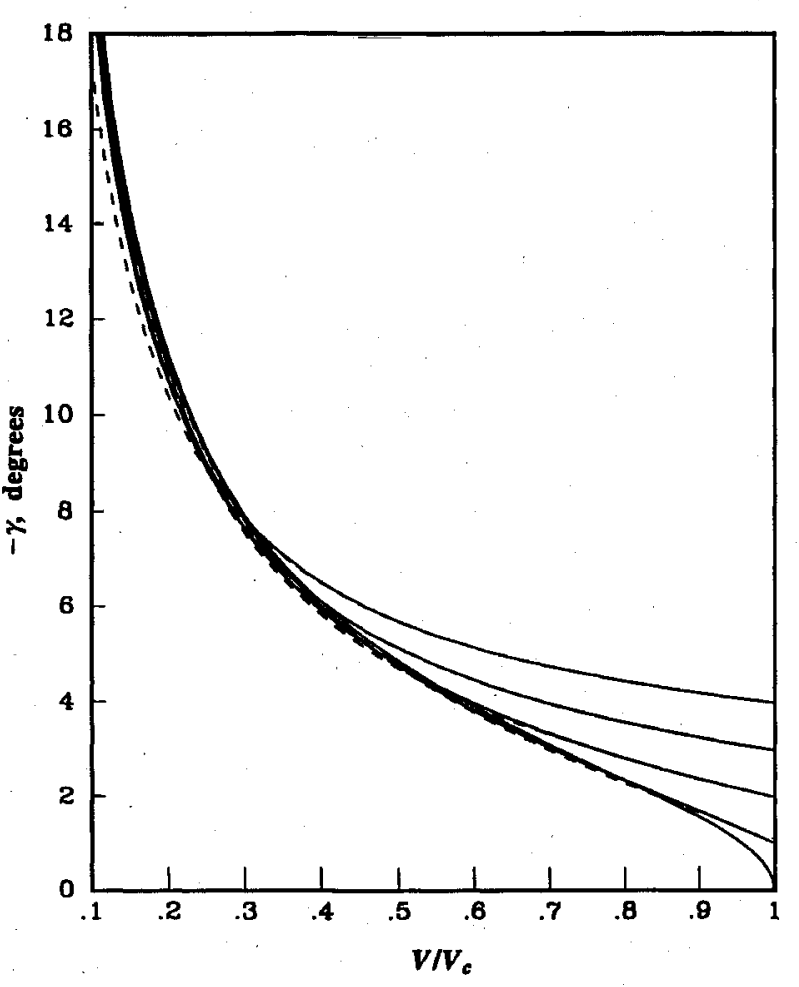

Fig. 4 Variation of the flight-path angle during ballistic entry at nearcircular speed. From bottom to top are trajectories with $-\gamma_{0}=0,1,2,3$, and $4 \mathrm{deg}$. Numerical solution $(-)$ and analytical solution $(-. .-)$ are nearly coincident.

Typically, for entry at circular speed, as $u$ decreases from unity to nearly zero, $x$ increases from the initial value $x=0$ to a final value of about $x_{f}=4.9$ at Mach 2. Ignoring $\left(2 / \beta r_{0}\right) \phi$ and using Eq. (15), Eq. (13b) can be rewritten in terms of $x$ (instead of $u$ ) as

$$
\frac{\mathrm{d} x}{\mathrm{~d} s}=Y
$$

This equation is then used to change the independent variable from $s$ to $x$ in the other equations:

$$
\begin{gathered}
\frac{\mathrm{d} Y}{\mathrm{~d} x}=\phi \\
\frac{\mathrm{d} \phi}{\mathrm{d} x}=\frac{e^{x}-1}{Y}
\end{gathered}
$$

Equations (17) are the modified Yaroshevskii's equations for ballistic entry. Elimination of $\phi$ yiclds a second-order, nonlinear equation for $Y$ :

$$
Y \frac{\mathrm{d}^{2} Y}{\mathrm{~d} x^{2}}=e^{x}-1
$$

Formal power-series solutions for $Y$ and $\phi$ can now be constructed satisfying the initial conditions at $x=0$ :

$$
\left.Y\right|_{x=0}=0,\left.\quad \phi\right|_{x=0}=c
$$

Through $\mathcal{O}\left(x^{3}\right)$, these are found to be $\mathrm{e}^{10}$

$$
Y=c x+(1 / 2 c) x^{2}+(1 / 12 c)\left[1-\left(1 / c^{2}\right)\right] x^{3}+\cdots
$$

and

$$
\begin{aligned}
\phi & =c+(1 / c) x+(1 / 4 c)\left[1-\left(1 / c^{2}\right)\right] x^{2} \\
& +(1 / 18 c)\left[1-\left(2 / c^{2}\right)+\left(2 / c^{4}\right)\right] x^{3}+\cdots
\end{aligned}
$$

These power-series solutions are only valid for large and moderate initial entry angles since, when $\gamma_{0}$ is small, $c$ is small and the radii of convergence of the series become very small. Thus, the solutions are limited to the initial portion of the ballistic entry. As proposed by Yaroshevskii, ${ }^{7}$ we remove the singularity at $c=0$ in the power series above by considering the first term in the series solution for $Y$ to be proportional to $x^{m}$. This gives the formal solutions to Eqs. (17) satisfying the initial condition $Y(0)=0, \phi(0)=0$ :

$$
\begin{gathered}
Y_{0}=(2 / \sqrt{3}) x^{\frac{3}{2}}\left[1+\frac{1}{3}(x / 4)+\frac{1}{6}(x / 4)^{2}+\cdots\right] \\
\phi_{0}=\sqrt{3} x^{\frac{1}{2}}\left[1+\frac{5}{9}(x / 4)+\frac{7}{18}(x / 4)^{2}+\cdots\right]
\end{gathered}
$$

Here, the subscript zero denotes the reference trajectory, that is, the grazing ballistic entry from circular speed with $\gamma_{0}=0$. These solutions are in good agreement with the numerically computed solutions, and in Fig. 4 the plot of Eq. (23) is indistinguishable from the numerical solution.

We now consider the perturbations from this grazing ballistic entry. If $\Delta Y$ and $\Delta \phi$ are the perturbations in $Y$ and $\phi$, then,

$$
Y=Y_{0}+\Delta Y, \quad \phi=\phi_{0}+\Delta \phi
$$

Substituting into Eqs. (17a) and (17b), we obtain

$$
\begin{gathered}
\frac{\mathrm{d} \Delta Y}{\mathrm{~d} x}=\Delta \phi \\
\frac{\mathrm{d} \Delta \phi}{\mathrm{d} x}=-\frac{\left(e^{x}-1\right) \Delta Y}{Y_{0}\left(Y_{0}+\Delta Y\right)}
\end{gathered}
$$

Eliminating $\Delta \phi$ between these two equations and putting

$$
y=\Delta Y / \sqrt{x}
$$

we obtain the nonlinear equation

$$
\frac{\mathrm{d}^{2} y}{\mathrm{~d} x^{2}}+\frac{1}{x} \frac{\mathrm{d} y}{\mathrm{~d} x}-\frac{y}{4 x^{2}}+\frac{\left(e^{x}-1\right) y}{Y_{0}^{2}\left(1+y \sqrt{x} / Y_{0}\right)}=0
$$

Using three terms of the solution (22) for $Y_{0}(x)$ and linearizing in $\Delta Y / Y_{0}$, we have

$$
\frac{\mathrm{d}^{2} y}{\mathrm{~d} x^{2}}+\frac{1}{x} \frac{\mathrm{d} y}{\mathrm{~d} x}+\frac{1}{4 x^{2}}\left(2+x+\frac{x^{2}}{4}\right) y=0
$$

It can be shown that, by a proper transformation of variables, this equation becomes a confluent hypergeometric equation. On the other hand, by neglecting the term $x^{2} / 4$ in the coefficient of $y$, we have a Bessel's equation of imaginary order. The handling of its solutions is rather cumbersome. Power-series solutions yield poor results when the entire range of interest of $x$ is considered. This is because, for small $x$, Eq. (28) is reduced to an Euler's equation with general solution:

$$
y=A \cos n \theta+B \sin n \theta
$$

where

$$
\theta=\ln x
$$

and

$$
n=\sqrt{2} / 2
$$

This solution clearly exhibits the oscillatory character of the perturbed motion. Therefore, we shall use a technique of construction of the complete solution of Eq. (28), as suggested by Boole. ${ }^{11}$

With the variable $\theta$ used as independent variable to replace $x$, the full equation becomes

$$
y^{\prime \prime}+n^{2} y+\frac{1}{4} e^{\theta} y+\frac{1}{16} e^{2 \theta} y=0
$$

where the prime denotes differentiation with respect to $\theta$. If we consider only the first two terms of Eq. (32), we have the solution (29) for Euler's equation. For the full equation, by considering $A$ and 
$B$ as functions of $\theta$, upon substituting into Eq. (32) and equating the coefficients of $\cos n \theta$ and $\sin n \theta$ to zero, we have the equations

$$
\begin{aligned}
& A^{\prime \prime}+2 n B^{\prime}+\frac{1}{4} e^{\theta} A+\frac{1}{16} e^{2 \theta} A=0 \\
& B^{\prime \prime}-2 n A^{\prime}+\frac{1}{4} e^{\theta} B+\frac{1}{16} e^{2 \theta} B=0
\end{aligned}
$$

We seek solutions for $A$ and $B$ in the form

$$
A=\sum_{m=0}^{\infty} a_{m} e^{m \theta}, \quad B=\sum_{m=0}^{\infty} b_{m} e^{m \theta}
$$

This results in the following recurrence formulas for the coefficients $a_{m}$ and $b_{m}$ :

$$
\begin{gathered}
a_{m}=-\frac{1}{4\left(2+m^{2}\right)}\left(a_{m-1}+\frac{1}{4} a_{m-2}\right) \\
+\frac{\sqrt{2}}{4 m\left(2+m^{2}\right)}\left(b_{m-1}+\frac{1}{4} b_{m-2}\right) \\
b_{m}=-\frac{\sqrt{2}}{4 m\left(2+m^{2}\right)}\left(a_{m-1}+\frac{1}{4} a_{m-2}\right) \\
-\frac{1}{4\left(2+m^{2}\right)}\left(b_{m-1}+\frac{1}{4} b_{m-2}\right)
\end{gathered}
$$

With $a_{0}$ and $b_{0}$ arbitrary, the complete solution for $y$, written in terms of the original variable $x$, is

$$
\begin{aligned}
y & =a_{0}\left\{f_{1}(x) \cos [(\sqrt{2} / 2) \ln x]-g_{1}(x) \sin [(\sqrt{2} / 2) \ln x]\right\} \\
& +b_{0}\left\{f_{1}(x) \sin [(\sqrt{2} / 2) \ln x]+g_{1}(x) \cos [(\sqrt{2} / 2) \ln x]\right\}
\end{aligned}
$$

where

$$
\begin{gathered}
f_{1}(x)=1-\frac{1}{12} x-\frac{1}{96} x^{2}+\frac{5}{12672} x^{3}+\frac{65}{1824768} x^{4}+\cdots \\
g_{1}(x)=\frac{\sqrt{2}}{12} x\left(1-\frac{3}{352} x^{2}+\frac{1}{38016} x^{3}+\cdots\right)
\end{gathered}
$$

Returning to the perturbation $\Delta Y=y \sqrt{x}$, its general solution takes the form

$$
\begin{aligned}
\Delta Y & =C \sqrt{x}\left\{f_{1}(x) \cos \left[(\sqrt{2} / 2) \ln \left(x / x_{0}\right)\right]\right. \\
& \left.-g_{1}(x) \sin \left[(\sqrt{2} / 2) \ln \left(x / x_{0}\right)\right]\right\}
\end{aligned}
$$

where now $C$ and $x_{0}$ are the arbitrary constants of integration. Subsequently, from Eq. (25a), we have for the perturbation in the flightpath angle

$$
\begin{gathered}
\Delta \phi=(C / 2 \sqrt{x})\left\{f_{2}(x) \cos \left[(\sqrt{2} / 2) \ln \left(x / x_{0}\right)\right]\right. \\
\left.-g_{2}(x) \sin \left[(\sqrt{2} / 2) \ln \left(x / x_{0}\right)\right]\right\}
\end{gathered}
$$

where

$$
\begin{aligned}
& f_{2}(x)=1-\frac{5}{12} x-\frac{5}{96} x^{2}+\frac{53}{12672} x^{3}+\frac{577}{1824768} x^{4}+\cdots(40 \mathrm{a}) \\
& g_{2}(x)=\sqrt{2}\left(1+\frac{1}{6} x-\frac{1}{96} x^{2}-\frac{29}{6336} x^{3}+\frac{101}{1824768} x^{4}+\cdots\right)
\end{aligned}
$$

The solutions (38) and (39) for $\Delta Y$ and $\Delta \phi$ represent the perturbations in altitude and in flight-path angle from grazing entry at circular speed as given by the reference solutions (22) and (23) for $Y_{0}$ and $\phi_{0}$. But these perturbed solutions have a singularity at the initial point, where $x=0$. Hence, to evaluate the constants of integration $C$ and $x_{0}$, we take $x=x_{0}$ as the point where these solutions become valid. Before that point, we use the Eqs. (20) and (21) for
$Y$ and $\phi$, assumed to be valid in the interval $x \in\left[0, x_{0}\right]$. Therefore, we use the definition (24) to write the condition at the point $x_{0}$ :

$$
\begin{gathered}
Y\left(x_{0}, c\right)=Y_{0}\left(x_{0}\right)+C \sqrt{x_{0}} f_{1}\left(x_{0}\right) \\
\phi\left(x_{0}, c\right)=\phi_{0}\left(x_{0}\right)+\left(C / 2 \sqrt{x_{0}}\right) f_{2}\left(x_{0}\right)
\end{gathered}
$$

These equations can be solved for the unknown constants $C$ and $x_{0}$. Explicitly, by eliminating $C$, we have the equation for $x_{0}$ :

$$
f_{2}\left(x_{0}\right)\left[Y\left(x_{0}, c\right)-Y_{0}\left(x_{0}\right)\right]=2 x_{0} f_{1}\left(x_{0}\right)\left[\phi\left(x_{0}, c\right)-\phi_{0}\left(x_{0}\right)\right]
$$

When the entry is slightly perturbed, $c$ is small and we can obtain an approximate solution for $x_{0}$ by putting

$$
x_{0}^{\frac{1}{2}}=(\sqrt{3} / 4) k c
$$

where $k$ is the new unknown to be found. Upon substituting into Eq. (42), we have the equation for $k$ to the order of $c^{4}$ :

$$
\begin{aligned}
k & =1+\frac{9}{32} k^{2}-\frac{15}{1024} k^{4}+\cdots+\frac{3 c^{2}}{64} k^{2}\left(1-k+\frac{11}{32} k^{2}+\cdots\right) \\
& +\frac{9 c^{4}}{8192} k^{4}\left(1-\frac{4}{3} k+\cdots\right)
\end{aligned}
$$

which can be solved through $\mathcal{O}\left(c^{2}\right)$ as

$$
k=1.671328785+0.113444530015 c^{2}+\cdots
$$

With these solutions, we have shown in Fig. 5 the perturbation $\Delta \gamma$ in the flight-path angle variable in terms of the speed ratio $V / \sqrt{ }\left(g_{0} r_{0}\right)$ for several small deflections in the entry angle. By referring to Fig. 4, it is now clear that when we add these perturbations to the reference solutions for grazing entry, accurate solutions for entry at small angles from circular speed are obtained.

In Fig. 6, we have plotted the solution $Y=Y_{0}(x)+\Delta Y$, now expressed in the dimensionless altitude $h$, vs the speed ratio $V / \sqrt{ }\left(g_{0} r_{0}\right)$ for several ballistic entries at small angles. Although the analytic solutions are obtained from the truncated system (17), the numerical results are obtained by integrating the complete equations (13). The analytic solutions are seen to be in excellent agreement with the numerical results.

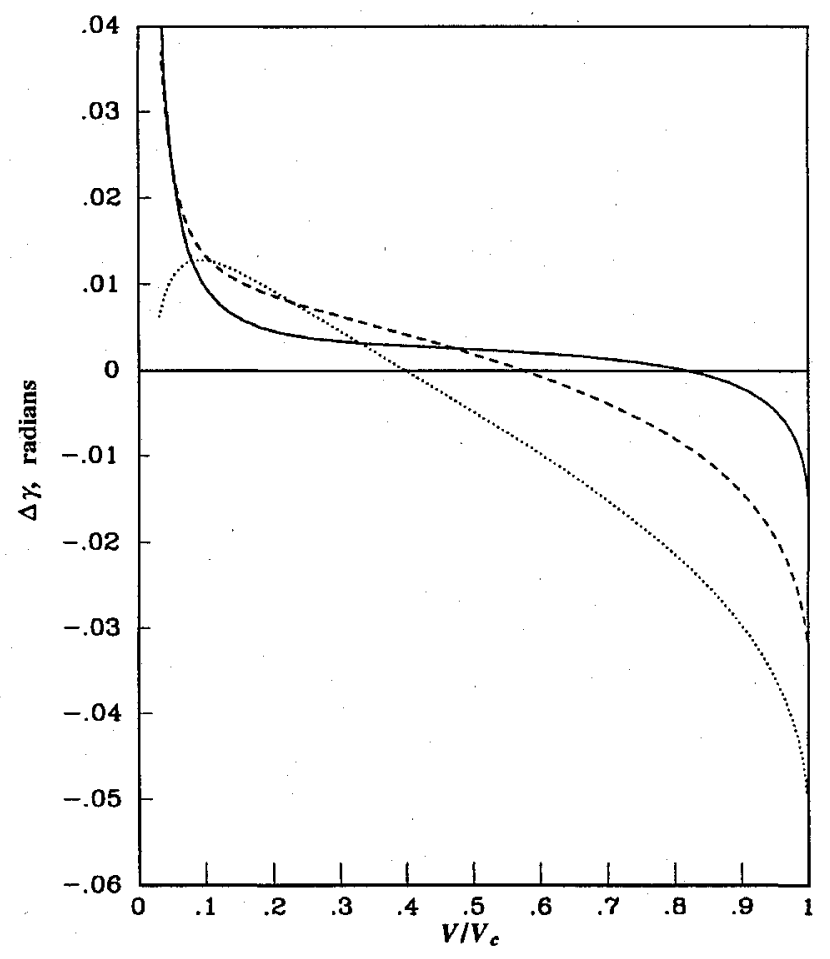

Fig. 5 Perturbation in the flight-path angle during ballistic entry at near-circular speed:,$--1 \mathrm{deg} ;,-\cdots,-2 \mathrm{deg}$; and $\cdots,-3 \mathrm{deg}$. 


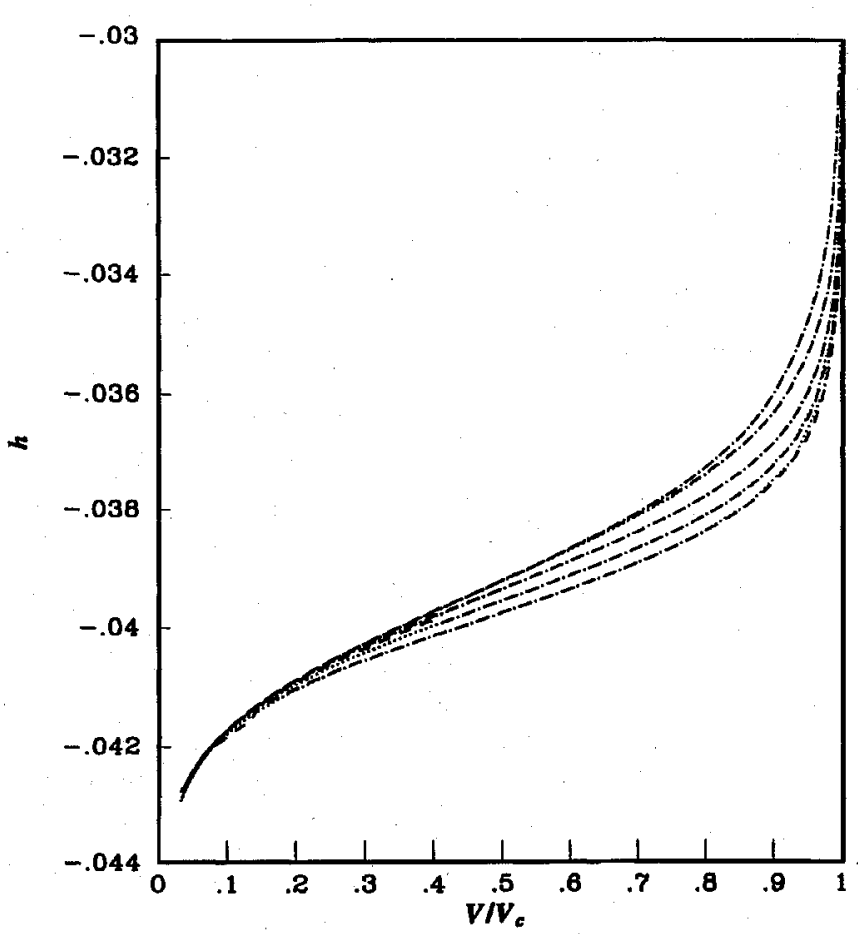

Fig. 6 Variation of the altitude during ballistic entry at near-circular speed. From top to bottom are trajectories with $-\gamma_{0}=0,1,2,3$, and $4 \mathrm{deg}$. Numerical solution $(-\cdots)$ and analytical solution $(\cdots)$ are nearly coincident.

It is seen in Figs. 4 and 6 that, with respect to the reference trajectory for grazing entry $\gamma_{0}=0$, in the perturbations for $\gamma_{0} \neq 0$, i.e., in the phugoid mode, the oscillations exhibit two crossover points where $\Delta Y=0$ and $\Delta \phi=0$, respectively. From Eq. (38), at the point $x$ where $\Delta Y=0$, we have

$$
\tan \left(\frac{\sqrt{2}}{2} \ln \frac{x}{x_{0}}\right)=\frac{f_{1}(x)}{g_{1}(x)}
$$

The solution for $x$ is a function of $x_{0}$ and therefore of the perturbed initial flight-path angle.

Although, for large values of $x$ (low speeds), $\Delta Y$ becomes large and negative [as can be seen from Eq. (38)], the ratio $\Delta Y / Y_{0}$ always decreases in magnitude, tending to zero at low speed. For this reason, we consider the perturbation in altitude to be stable.

Similarly, from Eq. (39) we compute the speed when $\Delta \phi=0$. This occurs when

$$
\tan \left(\frac{\sqrt{2}}{2} \ln \frac{x}{x_{0}}\right)=\frac{f_{2}(x)}{g_{2}(x)}
$$

Again, the critical speed depends on $x_{0}$, that is, on the perturbed initial flight-path angle. From the functional form of the analytic solution for $\Delta \phi$, Eq. (39), the perturbed flight path is a damped oscillation, although the period is rather long. As the speed decreases from the entry circular speed to nearly zero, $\Delta \phi$ passes through zero only once, at the critical speed found via Eq. (47). This speed is high when $\gamma_{0}$ is small. For large values of $x$ (low speed), $\Delta \phi$ tends to zero. Thus we consider the perturbation in the flight-path angle to be stable.

\section{Phugoid Motion in Equilibrium Glide}

The standard glide mode for the long-range, hypervelocity vehicle is the equilibrium glide, in which the acceleration normal to the flight path is zero. This leads to a very small and nearly constant flight-path angle during the descent. Hence, we consider Eq. (13c) with $\mathrm{d} \phi / \mathrm{d} s=0$. We then obtain the reference altitude (reference density) as a function of the speed as

$$
Y=\frac{2(1-u)}{\sqrt{\beta r_{0}}\left(C_{L} / C_{D}\right) u}
$$

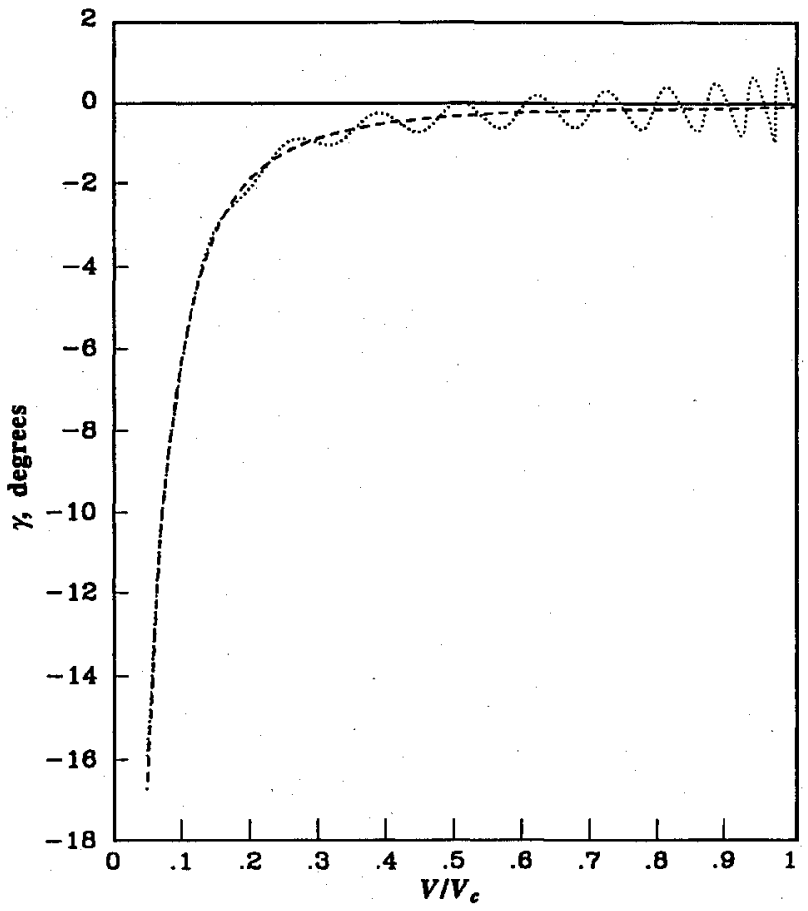

Fig. 7 Variation of the flight-path angle during gliding entry at nearcircular speed: -...-, equilibrium glide and... , perturbed.

Hence, in equilibrium glide the altitude decreases continuously as the speed decreases along the glide trajectory. To evaluate the flightpath angle, we combine Eqs. (13a) and (13b) to obtain

$$
\frac{\mathrm{d} u}{\mathrm{~d} Y}=-\frac{u}{\phi}+\left(\frac{2}{\beta r_{0}}\right) \frac{1}{Y}
$$

Using the solution (48) for $Y$ to evaluate $d Y / d u$, we have, upon substituting into Eq. (49) and solving for $\phi$,

$$
\phi=\frac{2 \sqrt{\beta r_{0}}(1-u)}{\left(C_{L} / C_{D}\right)\left[2+\beta r_{0} u(1-u)\right]}
$$

Equations (48) and (50) constitute the solutions for the reference equilibrium glide. For $Y=0, \phi=0$, the solutions lead to $u=1$ and we have the condition of circular orbital flight in the vacuum. To validate the use of these solutions, a very small, yet nonvanishing starting density $Y(0)$ is required. In addition, $u(0)$ and $\phi(0)$ must be such that the equations (48) and (50) are satisfied identically at the initial point.

Figure 7 presents the plot of the flight-path angle as a function of the speed ratio, $V / \sqrt{ }\left(g_{0} r_{0}\right)$, for equilibrium glide with $C_{L} / C_{D}=1.5$ (shown dashed). The numerical solution and the analytic solution from Eq. (50) are nearly identical down to a very low speed. Although it is possible to obtain higher-order solutions for equilibrium glide, the purpose of this work is the stability analysis of entry trajectories, so we use the first-order solution as the reference. Furthermore, in the denominator of Eq. (50), the term $\beta r_{0} u(1-u)$ is the dominant term. Hence, the variation of the flight-path angle as a function of the speed can be approximated by

$$
\phi=2 / \omega u
$$

where, by definition,

$$
\omega=\sqrt{\beta r_{0}}\left(C_{L} / C_{D}\right)
$$

The simplified solution (51) for a slow increase in the flightpath angle during the equilibrium glide is valid down to about a Mach 2 speed. This solution can be obtained directly by applying the equilibrium glide condition to the system (13) with the small gravity component neglected in Eq. (13b). Therefore, we use this 
truncated equation to write the system with $u$ as the independent variable replacing $s$ :

$$
\begin{gathered}
\frac{\mathrm{d} Y}{\mathrm{~d} u}=-\frac{\phi}{u} \\
\frac{\mathrm{d} \phi}{\mathrm{d} u}=\frac{\omega}{2 u}-\frac{1-u}{u^{2} Y}
\end{gathered}
$$

Taking the derivative of Eq. (53b) and using Eq. (53a), we obtain

$$
\frac{\mathrm{d}^{2} \phi}{\mathrm{d} u^{2}}=-\frac{\omega}{2 u^{2}}+\frac{2-u}{u^{3} Y}-\frac{1-u}{u^{3} Y^{2}} \phi
$$

By solving Eq. (53b) for $Y$, we have, upon substituting into Eq. (54), a nonlinear, second-order differential equation for $\phi$ :

$$
\begin{aligned}
& u(1-u) \frac{\mathrm{d}^{2} \phi}{\mathrm{d} u^{2}}-u \frac{\mathrm{d} \phi}{\mathrm{d} u}+\frac{\omega^{2}}{4} \phi=\frac{\omega}{2 u}-(2-\omega u \phi) \frac{\mathrm{d} \phi}{\mathrm{d} u} \\
& -\phi u^{2}\left(\frac{\mathrm{d} \phi}{\mathrm{d} u}\right)^{2}
\end{aligned}
$$

We have integrated this equation numerically, and the results duplicate the data from the integration of the original system (13). If the initial conditions are such that they verify the conditions (48) and (51), we have the equilibrium glide path. Any small initial deviations, either in the speed or in the flight-path angle or both, would result in a phugoid oscillation as shown by the dotted lines in Figs. 7 and 8 . Note that since we have near-grazing entry during the initial portion of the trajectory, the perturbation, say in the flight-path angle, may be much larger than the reference angle. This explains the subtlety in the linearizing process applied to the nonlinear equation (55). In this equation, if the reference solution (51) is used to evaluate the last two terms, considered as part of the forcing function to a linear equation with varying coefficients, we shall obtain $-8 / \omega^{3} u^{3}$. Therefore, one can neglect these terms to obtain the equation for the flight-path angle in the form

$$
u(1-u) \frac{\mathrm{d}^{2} \phi}{\mathrm{d} u^{2}}-u \frac{\mathrm{d} \phi}{\mathrm{d} u}+\frac{\omega^{2}}{4} \phi=\frac{\omega}{2 u}
$$

This is a hypergeometric equation with a forcing function. Its general solution is

$$
\phi=\phi_{c}+(2 / \omega u)
$$

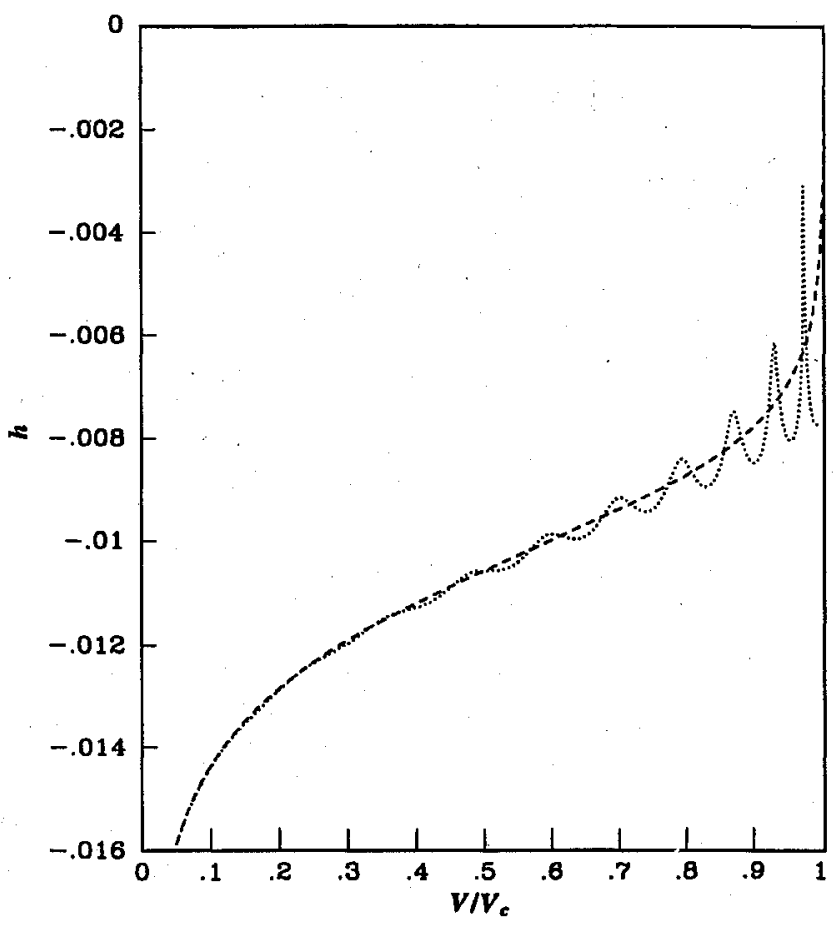

Fig. 8 Variation of the altitude during gliding entry at near-circular speed: $\cdots,-$, equilibrium glide and $\cdots$, perturbed. where $\phi_{c}$ is the complimentary function, the general solution of the homogeneous equation, and the additional term is a particular solution, which we have taken approximately as the equilibrium solution, since it has been shown that it is the solution to the system when $\mathrm{d} \phi / \mathrm{d} u \approx 0$. Instead of using the exact solution via hypergeometric functions, it is more enlightening to use an approximate integration to show the behavior of the phugoid in glide mode as a damped oscillation, which is evident in Figs. 7 and 8.

We introduce a new independent variable $\mu$ such that

$$
u=\cos ^{2} \mu
$$

As $u$ varies from 1 to $0, \mu$ increases from 0 to $\pi / 2$. In addition, the dependent variable is changed so that

$$
\phi=z / \sqrt{\tan \mu}
$$

to put the equation in the normal form

$$
\frac{\mathrm{d}^{2} z}{\mathrm{~d} \mu^{2}}+\left(\omega^{2}+\frac{1-4 \sin ^{2} \mu}{4 \sin ^{2} \mu \cos ^{2} \mu}\right) z=0
$$

Denote the varying term in the coefficient of $z$ as

$$
\frac{1-4 \sin ^{2} \mu}{4 \sin ^{2} \mu \cos ^{2} \mu}=\frac{4 u-3}{4 u(1-u)} \equiv f(u)
$$

When $u$ varies within its range of interest, from 0.95 to $0.05, f(u)$ decreases monotonically from 4.2105 to -14.7368 . On the other hand, $\omega^{2}$ is much larger. So we replace $f(u)$ by its mean value on the interval $0.05=u_{1} \leq u \leq u_{2}=0.95$ :

$$
\bar{f}=\frac{1}{u_{2}-u_{1}} \int_{u_{1}}^{u_{2}} f(u) \mathrm{d} u=-\frac{1}{1.8} \ln 19=-1.6358
$$

The coefficient of $z$ in Eq. (60) can be approximated by

$$
\bar{\omega}^{2}=\beta r_{0}\left(C_{L} / C_{D}\right)^{2}-1.6358
$$

Then an approximate solution for $z$ is obtained as

$$
z=A \cos \left[\bar{\omega}\left(\mu-\mu_{0}\right)\right]
$$

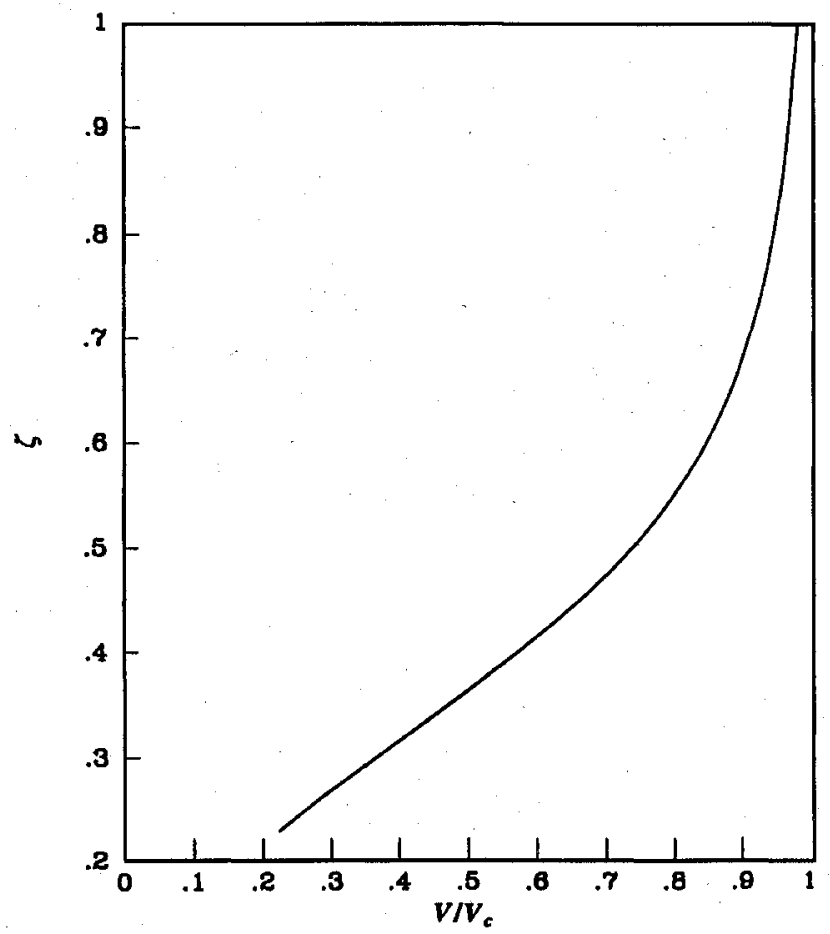

Fig. 9 Damping function in equilibrium glide; $\boldsymbol{u}_{e}=\mathbf{0 . 9 9 5 0 2 5}$. 
where $A$ and $\mu_{0}$ are arbitrary constants of integration. From the definition (59), the complementary function is approximated by

$$
\phi_{c}=A(\tan \mu)^{-\frac{1}{2}} \cos \left[\vec{\omega}\left(\mu-\mu_{0}\right)\right]
$$

In terms of the speed variable $u$, we now have the complete solution in the form

$$
\phi=\frac{2}{\omega u}+\frac{A u^{\frac{1}{4}}}{(1-u)^{\frac{1}{4}}} \cos \left[\tilde{\omega}\left(\mu-\mu_{0}\right)\right]
$$

It is clear that along the reference equilibrium glide $A=0$ and the flight-path angle decreases continuously. When a perturbation occurs, $\phi$ undergoes an oscillation with frequency $\bar{\omega}$ and damping provided by the function

$$
\zeta(u)=u^{\frac{1}{4}}(1-u)^{-\frac{1}{4}}
$$

As $u$ decreases from 0.95 to 0.05 , the damping function monotonically decreases from 2.0878 to its inverse value, 0.47897 . This function, rescaled so that the initial value is unity, is plotted vs the speed ratio $V / \sqrt{ }\left(g_{0} r_{0}\right)$ in Fig. 9. As compared to the variation in Fig. 7, it appears that the solution (67) correctly predicts the damping in the equilibrium glide mode.

As previously mentioned, $\mu$ varies from 0 to $\pi / 2$. Thus, over the complete range of the speed the number of oscillations with frequency $\bar{\omega}$ is computed by

$$
N=\bar{\omega} / 4
$$

As a numerical example, we take $C_{L} / C_{D}=1.5$ and evaluate $\bar{\omega}$ to obtain $N=11.25$. For glide entry perturbed from the equilibrium glide, the phugoid mode is a lightly damped oscillatory motion with a period much shorter than the orbital period.

Finally, the perturbation in the altitude is readily obtained from Eq. (53b) as

$$
\begin{aligned}
& \frac{1}{Y}=\frac{\omega u}{2(1-u)}+\frac{2}{\omega(1-u)}-\frac{A u^{\frac{5}{4}}}{4(1-u)^{\frac{9}{4}}}\left\{\cos \left[\bar{\omega}\left(\mu-\mu_{0}\right)\right]\right. \\
& \left.\quad+2 \bar{\omega} u^{\frac{1}{2}}(1-u)^{\frac{1}{2}} \sin \left[\bar{\omega}\left(\mu-\mu_{0}\right)\right]\right\}
\end{aligned}
$$

On the right-hand side of this solution, the first term gives the equilibrium glide solution, Eq. (48). The second term is very small, and the last term provides the damped oscillatory perturbation. It disappears at very low speed.

\section{Conclusions}

The phugoid motion in the critical case of grazing entry of hypervelocity vehicles into the Earth's atmosphere at circular speed has been investigated for the cases of ballistic entry and equilibrium glide. In both instances, the phugoid mode is a damped oscillatory motion with long period. Unsteady effects arising from the strong variations of the density and the speed during entry have been retained in the analysis. The new analytic solutions match the numerical solutions very well.

\section{References}

${ }^{1}$ Regan, F. J., and Anandakrishnan, S. M., Dynamics of Atmospheric ReEntry, edited by J. S. Przemieniecki, Vol. 33, AIAA Education Series, AIAA, Washington, DC, 1993.

${ }^{2}$ Chapman, D. R., "An Approximate Analytical Method for Studying Entry into Planetary Atmospheres," NASA TR R-11, 1959.

${ }^{3}$ Allen, H. J., "Motion of a Ballistic Missile Angularly Misaligned with the Flight Path upon Entering the Atmosphere and Its Effect upon Aerodynamic Heating, Aerodynamic Loads, and Miss Distance," NACA TN 4048, Oct. 1957.

${ }^{4}$ Allen, H. J., and Eggers, A. J., "A Study of the Motion and Aerodynamic Heating of Missiles Entering the Earth's Atmosphere at High Supersonic Speeds," NACA TN 4047, Oct. 1957.

${ }^{5}$ Mease, K. D., "Optimization of Aeroassisted Orbital Transfer: Current Statùs," Journal of the Astronautical Sciences, Vol. 36, Nos. 1/2, 1988, pp. 733.

${ }^{6}$ Loh, W. H. T., Dynamics and Thermodynamics of Planetary Entry, Prentice-Hall, Englewood Cliffs, NJ, 1963.

${ }^{7}$ Yaroshevskii, V. A., "The Approximate Calculation of Trajectories of Entry into the Atmosphere. I,' Kosmicheskie Issledovaniya, Vol. 2, No. 4, 1964, pp. 507-531 (in Russian).

${ }^{8}$ Etkin, B., "Longitudinal Dynamics of a Lifting Vehicle in Orbital Flight;" Joumal of Aerospace Sciences, Vol. 28, Oct. 1961, pp. 779-788.

${ }^{9}$ Vinh, N. X., and Dobrzelecki, A. J., "Non-Linear Longitudinal Dynamics of an Orbital Lifting Vehicle," NASA CR 1449, Oct. 1969.

${ }^{10}$ Longuski, J. M., and Vinh, N. X., "Analytic Theory of Orbit Contraction and Ballistic Entry into Planetary Atmospheres," Jet Propulsion Lab., Publication 80-58, California Inst. of Technology, Pasadena, CA, 1980.

${ }^{11}$ Boole, G., "On a General Method in Analysis," Philosophical Transactions of the Royal Society, 1844, pp. 225-282.

F. H. Lutze Associate Editor 